\section{An unusual maculopathy after routine cataract surgery}

Southampton Eye Unit, Southampton General Hospital, Tremona Road, Southampton, SO16 6YD, UK

Correspondence: M Costen, Tel: + 441482 604367; Fax: + 441482605327 . E-mail: Mtcosten@ doctors.org.uk

Received: 30 January 2006 Accepted in revised form: 9 August 2006 Published online: 24 November 2006

\author{
Abstract \\ Aims To report the presentation of apparent \\ acute and transient vitreomacular traction \\ causing reduction in visual acuity following \\ routine cataract surgery. \\ Methods A case series describing three \\ patients that developed acute and transient \\ vitreomacular traction following routine \\ cataract surgery by experienced surgeons. No \\ patients had any significant past medical or \\ ophthalmic history. The patients presented \\ shortly after surgery with reduction in visual \\ acuity and dramatic optical coherence \\ tomography (OCT) images suggesting \\ vitreomacular traction. \\ Results All three patients experienced \\ spontaneous resolution of symptoms and \\ clinical signs within the first few weeks after \\ surgery. Two patients experienced continued \\ mild metamorphopsia. \\ Conclusion To our knowledge, this is the first \\ documented report of such cases with \\ associated confirmation by OCT imaging. \\ Eye (2007) 21, 1416-1418; doi:10.1038/sj.eye.6702587; \\ published online 24 November 2006 \\ Keywords: cataract; surgery; vitreo-macular \\ traction; OCT \\ Cataract surgery is the most commonly \\ performed ophthalmic operation. The incidence \\ of sight-threatening complications is low, with \\ more than $85 \%$ of patients achieving $6 / 12$ or \\ better visual acuity in eyes with no ocular \\ comorbidity. ${ }^{1}$ We describe three patients who \\ presented with significantly blurred central \\ vision after apparently routine cataract surgery. \\ All three patients had a striking macular \\ appearance with optical coherence tomography \\ (OCT) imaging suggesting vitreomacular \\ traction. In all patients, clinical appearances \\ returned to normal spontaneously with an \\ improvement in visual acuity and some \\ improvement in symptoms.
}

Case reports

MTJ Costen, CPR Williams, S Asteriades and AJ Luff

\section{Case 1}

A 72-year-old Caucasian lady attended for left cataract surgery. Visual acuity had been measured at $6 / 9$ preoperatively and the patient had noticed no new visual symptoms since scheduling for surgery. The surgery was performed through a temporal clear corneal incision and was without incident. On waking the day after surgery, the patient noticed a 'dark cloud' in the central visual field of the left eye (LE). This failed to clear and the patient presented to eye casualty 2 days later.

The patient was otherwise fit and well and there was no relevant past ophthalmic history.

On examination, best-corrected visual acuity was Count Fingers LE. The eye was quiet with a well-centred posterior chamber intraocular lens (IOL) and an intraocular pressure of $16 \mathrm{~mm} \mathrm{Hg}$.

Fundoscopy demonstrated a spectacular appearance, with an irregular area of apparent increased choroidal visibility at the fovea, associated with localised retinal elevation and no clinical evidence of a posterior vitreous detachment (PVD) (Figure 1). OCT was difficult to interpret, but appeared to reveal gross foveal elevation, but no evidence of a full-thickness macular hole (Figure 2a).

One week later she reported that her vision was starting to improve, but that she was now noticing some distortion. On examination visual acuity was measured at $6 / 18$ with pinhole.

Surprisingly, on fundoscopy, the macula had returned to a normal appearance. There was still no clinical evidence of a PVD, and OCT confirmed a normal macular profile (Figure 2d).

Three weeks later, the patients' vision had improved to $6 / 6$ with pinhole, although the patient continued to report slight micropsia. 


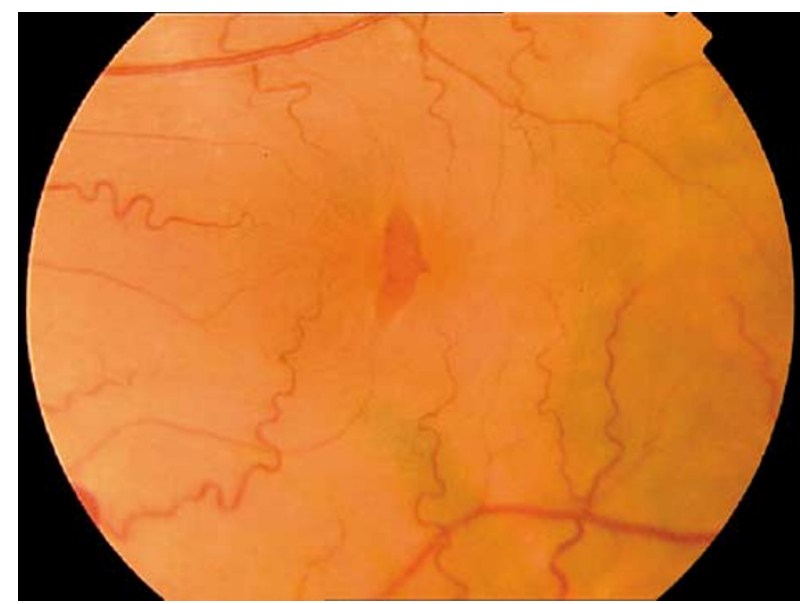

Figure 1 Clinical appearance of the macula in case 1. Gross macular thickening is evident with increased choroidal transmission at the fovea.

\section{Case 2}

A 59-year-old female presented 2 days after left cataract surgery with blurred vision. She had previously been highly myopic ( -8 dioptres spherical equivalent each eye) and had undergone successful right cataract surgery 6 months earlier.

Before surgery, best-corrected visual acuity was 6/6 right eye (RE) and 6/9 LE. She underwent routine phacoemulsification with IOL implant. On presentation she complained of a grey spot affecting central vision. Best-corrected visual acuity measured 6/60 in the LE. Fundoscopy revealed gross macular thickening, almost 'pouting' in appearance, with no evidence of PVD. OCT scanning demonstrated increased macular thickness, with its architecture distorted into an 'A'-shaped pattern (Figure 2b).

Over the following week the vision returned to normal with the patient only being aware of a slight difference in image size between the two eyes. Visual acuity measured 6/9 LE, 2 months after surgery, and repeat OCT scan was normal.

\section{Case 3}

A 66-year-old male presented 1 day after routine right phacoemulsification and IOL insertion with blurred vision. He had previously undergone successful cataract surgery to the LE and had no other relevant ophthalmic history. Preoperatively, best-corrected visual acuity in the RE had been measured as 6/9.

At presentation, vision was reduced to $6 / 24$. The eye was minimally inflamed, with one plus of cells in the anterior chamber. The macula showed marked thickening, with a 'puckered' appearance and pseudomacular hole. No PVD was present. OCT revealed
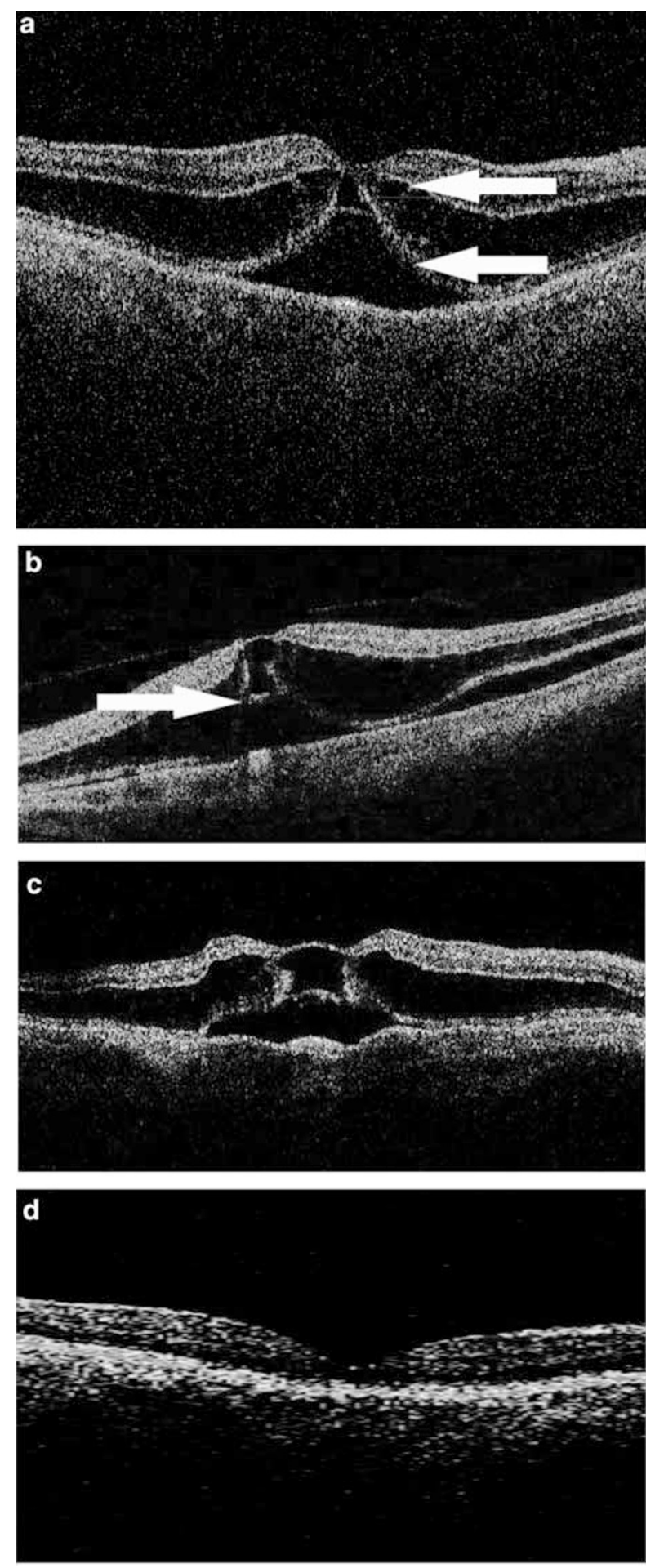

Figure 2 (a) OCT III ${ }^{\mathrm{TM}}$ (Carl Zeiss Meditech) imaging of the left macula in case 1 shows gross distortion of the normal foveal contour and apparent retinoschisis (arrows), but no evidence of a full-thickness macular hole. (b) OCT image of case 2 demonstrating similar appearance to case 1. The macula is distorted into an apparent ' $A$ ' shape on OCT (arrow). (c) OCT image of case 3. (d) Normal OCT appearance of left macula in case 1 a week later. 
markedly distorted macular architecture adopting an 'A'-shaped configuration (Figure 2c).

Vision gradually improved to $6 / 12$ RE over the next 2 months. OCT at this stage was normal, but he continued to experience micropsia and distortion of vision.

\section{Comment}

We present three cases of maculopathy presenting acutely and dramatically immediately after routine cataract surgery with rapid spontaneous recovery over the following few weeks. No patients were noted to have macular abnormalities preoperatively. Surgery was performed by three different surgeons with significant experience of cataract surgery. The surgery was without incident, and there were no difficulties encountered during surgery, such as excessive depth changes of the anterior chamber. All patients received standard postoperative topical medication comprising steroid and antibiotic drops.

The clinical appearances suggested very significant vitreomacular traction. OCT scanning in each case excluded the presence of a full-thickness retinal defect.

There are several possible explanations for this phenomenon. Vitreomacular traction causing foveal elevation has been reported following cataract surgery. ${ }^{2}$ Furthermore, full-thickness macular hole formation within a week of cataract surgery has been reported in a series of five eyes. ${ }^{3}$ This would appear to suggest the role of vitreo-foveal traction in the early postoperative period. ${ }^{4}$ There are also reports of re-opening of previously successfully closed macular holes following cataract surgery. ${ }^{5,6}$ However, there does not seem to be a strong temporal relationship between hole reopening and the time of cataract surgery in these reports. In none of these reports did spontaneous relief of vitreomacular traction or hole closure take place.

It seems likely that the cases we describe represent acute antero-posterior vitreo-macular traction after cataract surgery.
Following removal of the crystalline lens, the sudden volume change induced could conceivably be transmitted via the anterior hyaloid face to the posterior vitreous cortex. The spontaneous resolution in these cases may be the result of early postoperative return of normal ocular fluid dynamics.

It is possible that mild and transient vitreo-macular traction occurs commonly after cataract surgery. Further studies involving the use of OCT in the immediate postoperative period and B-scan ultrasonography would be needed to confirm this. If volume changes were responsible for this phenomenon, it is perhaps surprising that these findings do not occur more often.

Anecdotally some patients report the presence of floaters after cataract surgery, possibly owing to accelerated PVD development.

It is possible that in the patients we describe, there was an abnormally strong relationship between the macula and the posterior cortical vitreous, which resulted in symptomatic presentation after cataract surgery. As far as we are aware, this is the first report of such a phenomenon in the literature. Therefore we would like to suggest a name for this condition: 'A-sign maculopathy'.

\section{References}

1 Desai P, Minassian DC, Reidy A. National cataract surgery survey 1997-8: a report of the results of the clinical outcomes. Br J Ophthalmol 1999; 83: 1336-1340.

2 Falcone PM. Vitreomacular traction syndrome confused with pseudophakic cystoid macular edema. Ophthalmic Surg Lasers 1996; 27: 392-394.

3 Patterson JA, Ezra E, Gregor ZJ. Acute full-thickness macular hole after uncomplicated phacoemulsification cataract surgery. Am J Ophthalmol 2001; 131: 799-800.

4 Ameli N, Lashkari K. Macular hole following cataract extraction. Semin Ophthalmol 2002; 17: 196-198.

5 Paques M, Massin P, Santiago PY, Spielmann AC, Le Gargasson JF, Gaudric A. Late reopening of successfully treated macular holes. Br J Ophthalmol 1997; 81: 658-662.

6 Paques M, Massin P, Blain P, Duquesnoy AS, Gaudric A. Long-term incidence of reopening of macular holes. Ophthalmology 2000; 107: 760-765. 